\title{
Implementing a household joint activity-travel multi- agent simulation tool: first results
}

\author{
Thibaut Dubernet $^{1} \cdot$ Kay W. Axhausen ${ }^{1}$
}

Published online: 28 August 2015

(C) Springer Science+Business Media New York 2015

\begin{abstract}
In recent years, there has been a growing interest in the social dimension of travel, and how travel decisions are influenced not only by the global state of the transportation system, but also by joint decisions and interactions with social contacts. Such joint decisions are particularly important for a variety of behaviors: leisure activities are often performed with social contacts, and their location and timing is thus the result of a joint process; household "maintenance" tasks, such as grocery shopping or driving the children to school, are allocated to one single member; and of course joint (car) travel is the result of complex joint decisions. For this reason, the need to include household and friendship relationships into mobility simulation frameworks is becoming more and more obvious. This paper presents a game theoretic framework for the study of joint decision making, as well as an algorithm to search for approximate solutions of the resulting game. An implementation of this algorithm for the household case, using the MATSim software framework, is proposed and tested. The implementation of this algorithm is tested for the Zürich area, Switzerland, focusing on the possibility to perform joint trips. Those results allow to identify strengths and weaknesses of the approach, and directions for future work.
\end{abstract}

Keywords Multi-agent $\cdot$ Joint decisions $\cdot$ Household $\cdot$ Simulation

\section{Introduction}

In recent years, there has been a growing interest in the social dimension of travel, and how travel decisions are influenced not only by the global state of the transportation system, but also by joint decisions and interactions with social contacts-a clear sign for this interest being the regular workshops organized on this theme (Dugundji et al. 2008, 2011, 2012).

Kay W. Axhausen

axhausen@ivt.baug.ethz.ch

Thibaut Dubernet

thibaut.dubernet@ivt.baug.ethz.ch

1 Institute for Transport Planning and Systems (IVT), ETH Zurich, 8093 Zurich, Switzerland 
A very active field of research is the study and modeling of intrahousehold interactions and joint decision making, often using the classical random utility framework extended to group decision making. A classical way to cope with the possibly conflicting objectives of different members of the household is to specify a group level utility function. For instance, Zhang et al. $(2005,2007)$ develop a model where time for different activity types is allocated to household members, subject to time constraints (including equality of time participation in joint activities), using a group level utility function formulated as a multilinear combination of the individuals' utilities - that is, a linear combination of individual utilities and pair-wise product of individual utilities. Kato and Matsumoto (2009) use a linear combination of the utility functions of the household members as a group utility. The assumption behind this kind of models is the existence of "utility transfers": individuals accept to decrease their own utility if it allows to increase the utility of others by a certain fraction of their loss. Bradley and Vovsha (2005) focus on the "daily activity pattern" generation, with household "maintenance" tasks (e.g. shopping) allocation and possibility of joint activities. To do so, they assume a layered choice structure, choosing first a daily activity pattern for each member, and then assigning joint and maintenance activities. Gliebe and Koppelman (2005) also base their model on the daily activity pattern concept, choosing first a "joint outcome" (the sequence of individual and joint activities), and then an individual pattern for each household member. Those models rely on enumeration of the possible household level patterns. Gliebe and Koppelman (2002) also derived a constrained time allocation model, which predicts the time passed by two individuals in joint activities.

Rather than postulating a group level utility function, the models of those authors specify a special distribution for the error terms of the individuals. In this setting, the error term of the individuals are correlated so that the probability of choosing a given joint output is the same for all individuals. Ho and Mulley (2013) also estimate models in which members of the household perform choices constrained by the choice of a household level travel pattern. Their data, as well as the parameters of the models, show high joint household activity participation on weekends, and a high dependence of joint travel on trip purpose and household mobility resources. Those results highlight the importance of representing joint household decisions, in particular when going beyond the "typical working day". Vovsha and Gupta (2013) formulate a time allocation model for multiple worker households, which considers a positive utility for members of the household to be home jointly, as it makes joint activities possible. The estimation results show a significant influence of this kind of synchronization mechanism. Most models listed in this paragraph are specific to given household structures; in particular, separate models need to be estimated for different household sizes.

Household level decision processes have also been modeled with approaches which significantly differ from the classical random utility framework. Golob and McNally (1997) propose a structural equation model, which predicts time allocation and trip chaining based on the sociodemographics of a household. Golob (2000) also used a structural equation model to model the dependency of time allocations of the two heads (man and woman) of a household.

Another class of approaches, more oriented toward multiagent simulation than analysis, is the use of optimization algorithms to generate households plans. They handle the household scheduling problem by transforming it into a deterministic utility maximization problem. Contrary to the previously presented approaches, those alternatives do not lead to the estimation of a model against data. The first of those approaches was introduced by Recker (1995). By extending increasingly the formulation of the Pick-Up and Delivery Problem with Time Windows, a well studied combinatorial optimization problem, he 
formulates the problem of optimizing the activity sequence of members of a household as a mathematical programming problem. However, due to the complexity of the problem, the full problem cannot be solved exactly by standard operations research algorithms, and the activity durations are not part of the optimized dimensions. Chow and Recker (2012) designed an inverse optimization method to calibrate the parameters of this model using measured data. Also, the formulation from Recker (1995) was later extended by Gan and Recker (2008) to introduce the effects of within day rescheduling due to unexpected events. Another attempt to generate plans for households uses a genetic algorithm, building on a previous genetic algorithm for individual plan generation (Charypar and Nagel 2005; Meister et al. 2005). This algorithm optimizes sequence, duration and activity choice for a household, rewarding the fact that several members of the household perform the same activity simultaneously, in the way also used by Vovsha and Gupta (2013). Finally, Liao et al. (2013) formulate the problem of creating schedules for two persons traveling together as finding the shortest path in a "supernetwork", and solve this problem using exact shortest path algorithms. They however note that their model is specific to the two person problem, and that extension to larger numbers of agents may prove to be computationally expensive. All those approaches remained experimental, and were not integrated into multiagent simulation tools.

Another class of methods aiming at multiagent simulations consists of rule based systems, which use heuristic rules to construct household plans. Miller et al. (2005) develop such a model for household mode choice. The main difference with an individual mode choice model is the consideration of household level vehicle allocation. In their model, individuals first choose modes individually. If a conflict occurs, the allocation that maximizes the household level utility is chosen. The members which were not allocated a vehicle will fall back on their second best choice, and/or examine shared rides options. Arentze and Timmermans (2009) develop a rule base model which relies on a simulated bargaining process within the household. Though such models can easily represent complex decision processes, their calibration and validation is cumbersome.

Other authors have investigated the role of more general social networks on travel. One of the main incentives to conduct such studies comes from the continuous increase of the share of trips which are performed for leisure purpose (Schlich et al. 2004; Axhausen 2005). This fact represents a challenge for travel behavior modeling, as those trips are much more difficult to forecast than commuting trips: they are performed more sporadically, and data about those trips is much more difficult to collect-in particular concerning attributes of locations and events, which are needed to make models that do not only consist of random noise. Understanding better how destination choice for leisure trip is made is therefore essential to improve the accuracy of those forecasts.

Various studies have been conducted with the idea that an important factor in leisure trip destination choice, or activity duration choice, is the ability to meet social contacts. Examples of empirical work include Carrasco and Habib (2009), Habib and Carrasco (2011) or Moore et al. (2013). All those studies show a substantial influence of social contacts on the spatial and temporal distribution of activities. Based on an analysis of social network involvement and role, Deutsch and Goulias (2013) advocate considering the role individuals play in different social networks. Using latent class cluster analysis models to analyse the role of individuals in the various social networks they are involved in, they find that "the decision-making role of an individual can differ vastly across different social engagement types". Frei et al. (2012) demonstrated in a simulation experiment how considering social interactions in leisure location choice can help increase the accuracy of predicted leisure trip distance distribution. 
Another field of empirical research studies the spatial characteristics of social networks. For instance, Carrasco et al. (2008) studied the relationship between individual's socioeconomic characteristics and the spatial distribution of their social contacts. This kind of empirical work allows to specify and estimate models able to generate synthetic social networks, given sociode- mographic attributes and home location. An example of such a model, based on the results of a survey in Switzerland, can be found in Arentze et al. (2012). This kind of model is essential if one wants to include social network interactions in microsimulation model.

This integration of social networks in multiagent simulation frameworks has already been attempted by other authors. Due to their disaggregated description of the world, such models are particularly well suited to the representation of complex social topologies. Han et al. (2011) present experiments of using social networks to guide activity location choice set formation in the FEATHERS multiagent simulation framework. Using a simple scenario with 6 agents forming a clique, they consider the influence of various processes like information exchange and adaptation to the behavior of social contacts to increase the probability of an encounter. They do not, however, represent joint decisions, such as the scheduling of a joint activity. The same kind of processes have been investigated by Hackney (2009), using more complex network topologies, within the MATSim framework, used in this paper. Ronald et al. (2012) and Ma et al. (2011, 2012) present agent based systems which do integrate joint decision making mechanisms, based on rule based simulations of a bargaining processes. They are not yet integrated into any operational mobility simulation platform.

Building on all those ideas, the work presented in this paper aims at including explicit coordination of individuals in a multiagent simulation software framework. A game-theoretic model of joint decisions for daily planning is introduced, and a simulation algorithm implemented using the MATSim software framework. The process is designed to be able to handle complex social network topologies, but the current study focuses on intrahousehold ties only. The results of an implementation for intrahousehold ridesharing for a scenario for the Zurich area, Switzerland, are analyzed. Comparing the results of this scenario with travel diary data, limitations of the current implementation are identified, and directions for future work are sketched.

\section{Method}

We present here a simulation framework able to represent joint decisions, that is, behavior requiring explicit coordination between individuals-such as shared rides, social activities or intrahousehold task allocation. The basic idea is that social contacts will take such a joint decision if it results in an improvement in the satisfaction of all participants. The modeling of the interaction of individuals with possibly conflicting objectives has been the subject of game theory for decades, making this theoretical framework particularly well suited for the problem at hand.

A game theoretic view of transportation systems has indeed been popular since the seminal work of Wardrop (Wardrop 1952). The essential idea behind it is to see the transportation system as a set of shared resources (road space, public transport vehicle seats, etc.), for which individuals compete, individuals in the population trying to maximize their own satisfaction, given the resources left available by others. Game theory studies solution concepts for such strategic interactions. A game theoretic solution concept 
is a definition of which states are equilibria, that is, stable under assumption of rationality-a state being considered stable if no agent/player has an incentive to change its behavior. The static, trip-based approach of Wardrop has been refined and extended with time. In particular, the equilibrium idea can be pretty naturally transfered to the activity based framework: individuals do not just try to optimize their trips, but their whole day. This is in particular the approach of the MATSim software framework (Axhausen 2006; Nagel and Flötteröd 2009).

Most solution concepts in transportation are akin to the Nash equilibrium: a state where no individual can improve its satisfaction by unilaterally changing its behavior. This kind of solution concept does not allow to represent joint decisions. This can be illustrated by a classical game, called the House Allocation Problem (Schummer and Vohra 2013). This game consists of $n$ players and $n$ houses. Moreover, each player has its individual ordering of the houses, from the most preferred to the least preferred, and players prefer being allocated alone to any house rather than in the same house as somebody else. The strategy of a player is the house it chooses to live in.

An interesting feature of this game is that any one-to-one allocation of players to houses is a Nash Equilibrium: no player can improve its payoff by unilaterally changing its strategy, as it would require choosing an occupied house. This result however contradicts basic intuition about the stability of such an allocation. In this particular case, a more realistic solution concept is the Absence of Blocking Coalition: given a one-to-one allocation of houses to players, a blocking coalition is a set of players which could all be better off by re-allocating their houses among themselves. It is to be noted that both solution concepts correspond to rational agents, i.e. agents having a preference ordering over outcomes. The only difference lies in the degree of communication which is allowed.

In the activity-based framework, this solution concept naturally becomes what we phrase the Absence of Improving Coalition solution concept. An improving coalition for a given allocation of daily plans is a set of social contacts that can all be better off by simultaneously changing their daily plan-for instance by switching from separate dinners at home to a joint dinner at a restaurant. The simulation of joint decision consists in the search of an allocation of daily plans without such coalitions.

This definition relies on a specification of individual preference ordering over the set of possible outcomes. This preference ordering is defined using a utility function, which values how much an individual is satisfied with its day. It uses a linear disutility of travel time, and a logarithmic utility of time passed performing activities, as proposed by Charypar and Nagel (2005). Different parameters can be defined for each mode/activity type.

In this formulation, the utility of a plan takes the form of a sum of the activity of performing activities and of the disutility of traveling:

$$
F={ }_{t \in \mathrm{T}} U_{\mathrm{act}, \mathrm{t}}+{ }_{a \in A} \varepsilon_{a}+{ }_{c \in C} U_{\mathrm{tog}, \mathrm{c}}+{ }_{i=2}^{n} U_{\text {trav }}\left(\operatorname{loc}_{i-1}, \operatorname{loc}_{i}\right)
$$

The utility for an activity type $t \in \mathrm{T}$ is $U_{\text {act,t }}=U_{\text {dur,t }}+U_{\text {short.dur,t }}$ with:

$$
\begin{gathered}
U_{\text {dur }, \mathrm{t}}= \begin{cases}\beta_{\mathrm{dur}} \cdot d_{t}^{*} \cdot \ln \frac{d_{t}}{d_{0, t}} & \text { if } d_{t}>d_{0} \\
0 & \text { otherwise }\end{cases} \\
U_{\text {short.dur }, \mathrm{t}}=\beta_{\text {short.dur }} \cdot d_{\text {short } \mathrm{t}}
\end{gathered}
$$


where $d_{t}^{*}$ is the "typical duration" for activity type $t, d_{0, \mathrm{t}}$ is the "minimal duration" for activity type $t, d_{\mathrm{t}}$ is the cumulated time passed performing activities of type $t, d_{\text {short, }}$ is the sum, for all activities of type $t$ shorter than $d_{\text {short }}$, of the differences with $d_{\text {short }}$.

In addition to this deterministic part, each activity $a \in \mathrm{A}$ has a random utility $\varepsilon_{\mathrm{a}}$, to representindividual taste variations. This number is randomly generated using a seed composed from the position of the activity in the plan, the agent and the location: for a given combination of those parameters, the value of this random term will be the same (Horni 013). Those terms follow an Extreme Value Distribution, as is traditional in choice models.

$U_{\text {tog,c, }}$, the utility of time passed with social contact $c \in \mathrm{C}$, can take the same form as the activity scoring, or a linear form.

The utility of traveling with mode $m$ is simply a linear function of travel time:

$$
U_{\text {trav }, m}\left(t_{\text {trav }}\right)=\alpha_{\mathrm{m}}+\beta_{\text {trav }, \mathrm{m}} \cdot t_{\text {trav }} .
$$

To search for states satisfying a game-theoretic solution concept with a search space too big for exhaustive search, co-evolutionary algorithms are particularly well suited. Popovici et al. (2012) provides a thorough introduction to this family of algorithms. Those algorithms work by decomposing a solution into different parts, which are co-evolved, the fitness of a partial solution being evaluated by interaction with other sub-solutions-a process naturally game-theoretic (Ficici 2004).

The definition of such an algorithm for the simulation of a transportation system is natural: each agent, representing an individual, performs an evolutionary algorithm to optimize its daily plan. The fitness of a daily plan is measured after an execution step, where agents concurrently execute their daily plans in a simulated transport system, influencing each other's fitness by road congestion, public transport vehicle crowding, interaction at social activities, etc. Those scores are then used to guide evolution towards the proper solution concept.

This results in the process illustrated on Fig. 1, which steps are described in more details below.

\section{Initial demand}

All agents have an initial daily plan, which will serve as a starting point for the iterative improvement process. Some characteristics of the plans are left untouched during the simulation, and should therefore come from data or external model. This is typically the case of long term decisions, such as home and work locations, or decisions involving a larger time frame than a single day (e.g. do the weekly shopping or not). The combination of those initial plans and of the mutation operators applied to the plans (see "Replanning" section) define the universal choice set of the agents.

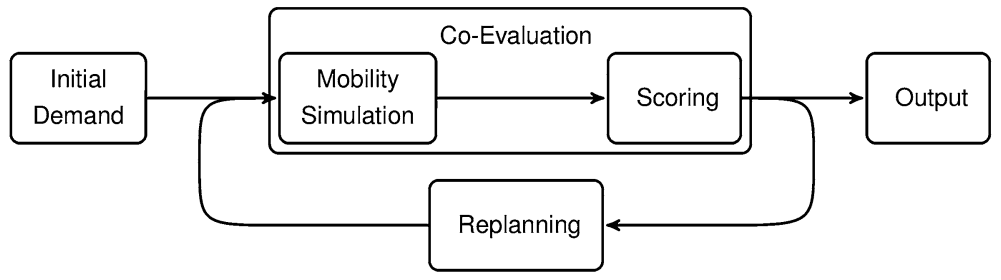

Fig. 1 The MATSim co-evolutionary process 


\section{Mobility simulation}

Plans of all agents are executed concurrently, to allow estimating the influence of the plans of the agents on each other. This step uses a queue simulation to simulate car traffic, which gives estimates of the congested travel time. Public transport vehicles are also simulated according to a schedule, and agents can experience delays if a vehicle becomes full, forcing them to wait for the next vehicle (Rieser et al. 2010). Together with the next step, this step constitutes the evaluation stage of the co-evolutionary algorithm.

\section{Scoring}

The information from the simulation is used to assign a score to each individual plan, using the utility function described above.

This gives the score from a single interaction. The fitness of the daily plan can then be updated, as $(1-\alpha) f_{\text {old }}+\alpha f_{\text {new }}$, with $\alpha \in[0.5,1]$ being the learning rate. The lower the learning rate, the more the fitness of a plan will be close to a successive average of fitnesses over the evaluated interactions. While this is consistent with the hypothesis that individuals react to the expected state of the transport system, most applications use a learning rate of 1 , which results in more reactive agents, and thus faster convergence. Nagel and Flötteröd (2009) define convergence as reaching a state where "agents draw from a stationary choice distribution such that the resulting distribution of traffic conditions re-generates that choice distribution". In practice, convergence is considered reached when the macroscopic statistics (such as mode shares and traffic counts) remain stable between iterations. For a more in-depth discussion of multi-agent equilibrium concepts, the interested reader might want to consult Nagel and Flötteröd (2009) or Meister (2011).

\section{Replanning}

This step actually groups two of the important components of co-evolutionary algorithms: (i) selection of the interactions for evaluation, and (ii) application of the evolutionary operators (selection and mutation).

Popovici et al. (2012) note that in co-evolutionary algorithms, "decisions need to be made as to what interactions should be assessed and how the outcomes of those interactions should be aggregated to give individual fitnesses". In the case of the activity scheduling problem studied here, the interactions (i.e. which daily plans are concurrently executed) should be selected such that (a) agents have time to adapt to the traffic patterns, (b) agents are able to estimate the influence of joint decisions on their score, while (c) keeping the number of computationally expensive mobility simulation steps as low as possible. (a) is achieved by letting a large proportion of agent select a past plan. (b) is achieved by including joint plans constraints. A joint plan is a set of individual plans executed simultaneously. Different copies of the same individual plan can be part of different joint plans-for instance an agent might go to the same restaurant alone, with members of its household or with a group of friends. The score of the different copies will take into account the influence of the joint plan to which it pertains. Those joint plan constraints are included using heuristic rules, applied after mutation operators are applied, and are classified between strong and weak constraints-weak constraints are considered when selecting plans for execution, but are allowed to be broken when merely selecting plans for mutation. They are then part of the evolution process. In the current application, 
the heuristic rules consist in joining newly created plans with joint trips (strong) or with leisure activities at the same location at the same time (weak).

To allow handling joint plans, agents need to be handled in groups. This is straightforward for households: all agents of a same household are always handled as a single group. For more general social networks, agents are handled with all agents with whom they have a joint plan, plus some social contacts with whom new joint plans can be created. As this paper focuses on intra-household interactions, details of how to group agents for generic social networks are left aside here. The interested reader can find a more general description of this replanning process, defined in terms of generic social network topology, in Dubernet and Axhausen (2013).

For each group, two actions are then possible. For most groups, an allocation of existing plans, fulfilling the joint plans constraints, is selected for execution. Based on plan scores, randomized by adding an extreme value distributed error term, an allocation without improving coalitions is searched for by an algorithm inspired by the "Top Trading Cycle" algorithm used for the House Allocation Problem (Schummer and Vohra 2013).

For the other groups, a plan allocation is selected and copied. The copied plans then undertake mutation, to make the agents explore new alternative joint plans. What kind of mutation is performed determines which alternative plans will be tried out by the agent. Mutation operators include:

- least cost path routing, given the congested travel times from the last execution step

- departure times random mutation

- mutation of modes at the subtour level, taking into account chaining constraints

- creation of shared rides, by joining a car trip (driver) with a non-chain based mode trip for another agent (the passenger). Currently, the process does not generate pure escorting trips.

- switching the sequence of two activities

- choice of a joint leisure location, by choosing a random location inside of the intersection of approximate space-time prisms for the participating agents

Agents have a limited memory size, keeping at most 3 plans per joint plan composition, and 10 plans in total. If this limit is exceeded, one should keep the plans which have the highest probability to create improving coalitions, that is, to be preferred to the other plans in the agent's memory. To this end, a lexicographic ordering is used: the process removes the joint plan which maximizes the number of individual plans which are the worst of the agents' memories. If several joint plans have the same number of worst plans, the process chooses among them the joint plan which maximizes the number of second worst plans, and so on until the "worst" joint plan is unique. When the overall maximum number of plans in the memory of an agent is reached, the worst individual plan for this agent is removed along with plans of other agents of the same joint plan. Each agents keeps at least one plan not part of a joint plan, as there may otherwise not be any state without blocking coalitions. Agents are parsed in random order, to avoid the emergence of "dictators" over iterations, whose worst plan would always be removed, even if it is the only "bad" plan of a joint plan.

Though those selection operators seem to be in accordance with the chosen solution concept, it is difficult, if not impossible, to prove that the process will actually converge towards the searched state. As noted by Ficici et al. (2005), when they perform a theoretical analysis of different selection methods in a co-evolutionary context, "Co-evolutionary dynamics are notoriously complex. To focus on our attention on selection dynamics, we will use a simple evolutionary game-theoretic framework to eliminate 
confounding factors such as those related to genetic variation, noisy evaluation, and finite population size". Those "confounding factors" can however not be eliminated from an actual implementation of a co-evolutionary algorithm, and rigorously proving that a given algorithm actually implements a specific solution concept is very tedious, if not impossible.

With iterations, agents build a choice set of daily plans that becomes better and better given the actions of the other agents. However, the presence of a large portion of agents with plans resulting from random mutation creates noise, not only for the analyst looking at the output of the simulation, but for the agents themselves when they compute the score of their plans. To solve this issue, when the system reaches a stable state, agents stop performing mutation, and only select plans from their memory for 100 iterations, using the absence of improving coalition with randomized scores. This ensures that the selected plans are the result of a behavioral model, rather than the result of random mutation operators.

\section{Output}

Once convergence was reached, data from the last iteration (daily plans and events from the mobility simulation) are used for analysis.

\section{Validation}

This section presents the results of a run of the model for the region of Zurich, Switzerland, and analyses how the results compare to the survey data.

\section{Scenario}

For this validation, a scenario for the Zurich area, Switzerland, was used. The scenario is composed of the following elements:

1. Population the initial demand comes from the full-Switzerland scenario described by Meister et al. (2010). It was generated by allocating activity chains from the national travel survey from the years 2000 and 2005 to records from the national census 2000, which is a $100 \%$ survey of the Swiss population, containing in particular information on home location at the hectare level and work location at the municipality level, as well as household membership information. The agents are grouped according to the household information from the census, and only the households having at least one member performing a trip passing less than $30 \mathrm{~km}$ from the Bellevue Place, in the center of Zurich, are kept. A sample of $10 \%$ of those households is used for the simulation. This results in a scenario containing 206,943 agents, grouped in 88,439 households, and performing a total of 788,931 trips. This rather old scenario is used because from 2010 on, the Swiss census contains detailed information only for a $2.5 \%$ of the Swiss population, and hence does not allow to reconstruct households as easily. Though this approach is not applicable to most recent data, it is sufficient for the purpose of this paper. The generation of a synthetic population, including household structure, from the most recent data is in progress (Müller and Axhausen 2014).

2. Network a planning network is used, from the National Transport Model (Vrtic et al. 2003). It models the Swiss network at a coarse resolution, as well as the major arterials in the neighboring countries. It allows faster runs than a navigation network. 
3. Public transport the public transport schedule from the Cantonal Transport Model is used to get realistic travel time estimates (Amt für Verkehr, Volkswirtschaftsdirektion Kanton Zürich 2011).

4. Facilities the "facilities" contain the information about opening times for different activity types, and roughly correspond to buildings. Data comes from the federal enterprise census 2001.

Due to the evolving code base, the parameters of the scoring function which were calibrated for previous studies did not give satisfying results anymore. Therefore, the parameters were adjusted so as to obtain reasonable results. Due to the duration of a single simulation, calibrating a scenario is a time-consuming process. The aim of the study described herein being to test the behavior of the model rather than producing accurate forecasts, it was not attempted to get a perfect fit, but rather to obtain reasonable values-a more thorough calibration is in progress for a scenario build with most recent data, which however does not contain household information. Each agent have private preferences about leisure locations, modeled by an agent-specific Gumbel distributed error term for each location, using the approach of Horni (2013).

Two variants of the scenario are run: one with, and one without giving the possibility to the agents to adjust location of leisure activities and sequence of the activities. We will refer to those two variants as LOC and NOLOC, respectively.

The replanning modules used for those runs, as well as their weights, are presented in Table 1. For each household, at each iteration, a replanning module is selected with a probability proportional to the weights of the replanning module. This process in run for 1500 iterations. During those iterations, agents concurrently improve the quality of the

Table 1 Probability of the different replanning modules

\begin{tabular}{|c|c|c|}
\hline Module & Description & Weight \\
\hline Logit-like & Selection Selects past plans using Gumbel-distributed scores & 2 \\
\hline Time allocation mutation & $\begin{array}{l}\text { Randomly mutates activity end times. It adds or removes a random } \\
\text { amount to all activity end times in a plan, within a range which } \\
\text { decreases with iterations, from }[-2.5 h ;+2.5 h] \text { at the } \\
\text { beginning to }[-0.5 h ;+0.5 h] \text { from iteration } 750 \text { on. The big } \\
\text { mutation range at the beginning helps synchronizing plans of co- } \\
\text { travelers; the smallest range at the end allows a finer tuning of } \\
\text { departure times }\end{array}$ & 1 \\
\hline Subtour mode mutation & $\begin{array}{l}\text { Changes randomly the mode of all trips of a subtour. It considers } \\
\text { car availability (i.e. the combination of driver's license and car } \\
\text { ownership) and trip chaining constraints: subtours with chain- } \\
\text { based modes (car and bike) must be anchored at home or in a } \\
\text { subtour of the same mode. Subtours containing one or more joint } \\
\text { trips are not modified }\end{array}$ & 1 \\
\hline Re-routing & $\begin{array}{l}\text { Compute new routes for all trips in the plan, using a least-cost path } \\
\text { algorithm based on the travel times observed in the previous } \\
\text { iteration }\end{array}$ & 1 \\
\hline Joint trip mutation & Inserts or remove joint trips randomly & 1 \\
\hline Sequence mutation & $\begin{array}{l}\text { Swaps the position of two randomly chosen activities in the plan } \\
\text { This module is only activated in LOC }\end{array}$ & 1 \\
\hline Joint location choice & $\begin{array}{l}\text { Randomly mutates leisure locations, using a household-level } \\
\text { choice set } \\
\text { This module is only activated in LOC }\end{array}$ & 1 \\
\hline
\end{tabular}


plans in their choice set, given the behavior of the others. For 100 additional iterations, all modules except Logit-like plan selection are deactivated: agents just choose their behavior from their choice set, updating the score of the plans to the new behavior of others.

The comparison of the results of those runs with the data from the national travel survey 2005 are discussed in the next section. For most of the results, only the trips having their origin and their destination within a radius of $20 \mathrm{~km}$ around the Bellevue Place are considered, due to the way the scenario was generated (see above). The radius is smaller than the $30 \mathrm{~km}$ used as a criterion during scenario generation, to avoid border effects.

\section{Results}

Figure 2 shows the final mode shares per distance class for all the scenarios, as well as in the National Travel Survey 2005, considering only the trips having their origin and their destination $20 \mathrm{~km}$ from Bellevue Place. The length of a trip is defined as the sum of the crow-fly distances of all stages, as it is the only statistic available for all modes and both from the simulation and the National Travel Survey. The scenario in general contains too few short trips compared to the National Travel Survey, though letting agents choose their leisure destination helps improve this statistic. Overall, the fit for all modes but bike is rather good-though the fact that joint travel is considered only within the household makes difficult to assess whether agents travel too much together.

Figure 3 shows the distribution of trip distances in the different scenarios and the National Travel Survey 2005. Outliers are not shown. "driver" corresponds to driving a car with a passenger. As above, trip distance is estimated using the sum of stages crow-fly distances. Trip length for bike and walk are overestimated, probably due to the linear form

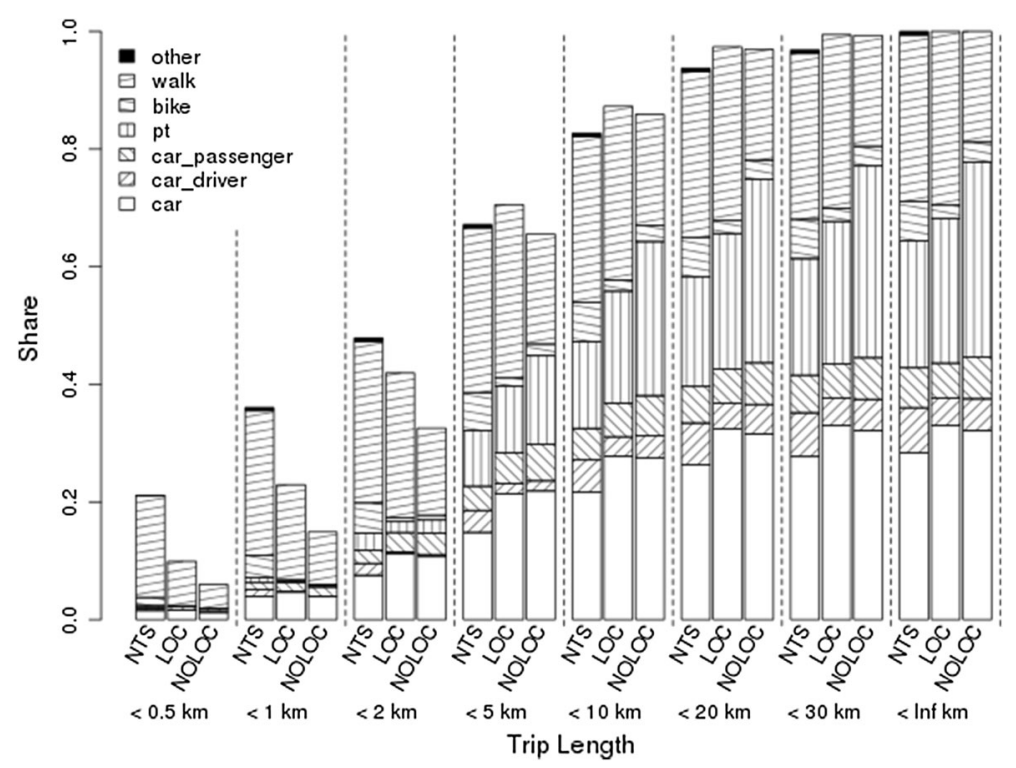

Fig. 2 Mode share per distance class, in the National Travel Survey (NTS) and simulations, with (LOC) and without (NOLOC) leisure destination adaptation 


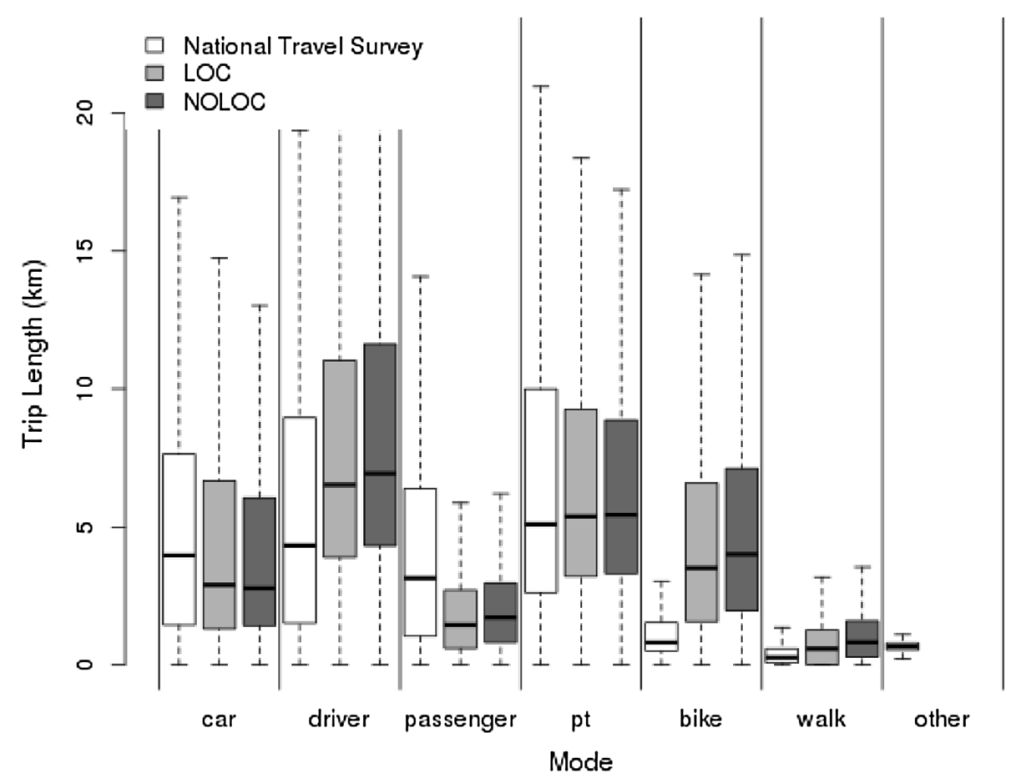

Fig. 3 Trip length distribution per mode, in the National Travel Survey and simulations, with (LOC) and without (NOLOC) leisure destination adaptation

of the disutility of travel time for those modes (i.e. it does not consider tiredness), and to the under-representation of trips under $2 \mathrm{~km}$ in the scenario. When driving somebody, agents travel a bit too much, whereas trips being performed as a passenger are much shorter than in the National Travel Survey. This indicates that drivers tend to perform too large a part of their trip alone in the car. This might be due to a wrong distribution of purposes, as will be seen in Fig. 4: if when dropping the children to school on the way to work, the joint part might be rather short compared to the full trip, for other purposes, such as leisure, individuals might do their trip between the same origin and destination.

Figure 4 presents the share of the "car passenger" mode, from activity type to activity type, in the activity chains extracted from the National Travel Survey, as well as in the simulations. Figure 4(c) uses data from all Switzerland, due to the small sample size; the other figures consider only the trips with origin and destination within a $20 \mathrm{~km}$ radius around the Bellevue Place. In the National Travel Survey, the share of the car passenger mode is particularly high among trips with leisure purpose, indicating a high share of joint activities. Simulations do not exhibit this pattern. Several reasons may explain this fact: (a) only intra-household ties are considered, (b) the initial activity schedules for household members were generated independently, and (c) pure serve passenger tours are not considered. Interestingly, the share of being a passenger between home and school is very high, as should be expected in households - even too high, probably linked to the fact that the global mode share of being a passenger is close to the observed one, even if only intrahousehold joint trips are considered. Those trips emerge from the constraints specific to households, namely the fact that children do not have a car, and that the daily tours of all members of the household originate and end at home, making it easy to join trips. The willingness to help (represented by the positive constant for being a driver) then makes those trips more attractive than their individual counterpart, for both driver and passenger. 
(a) Scenario LOC

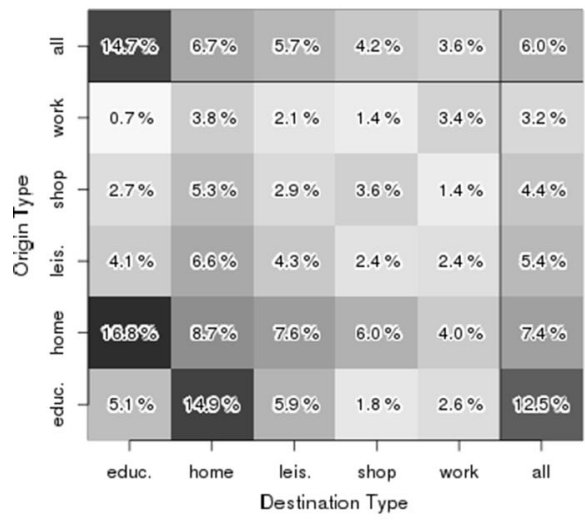

(b) Scenario NOLOC

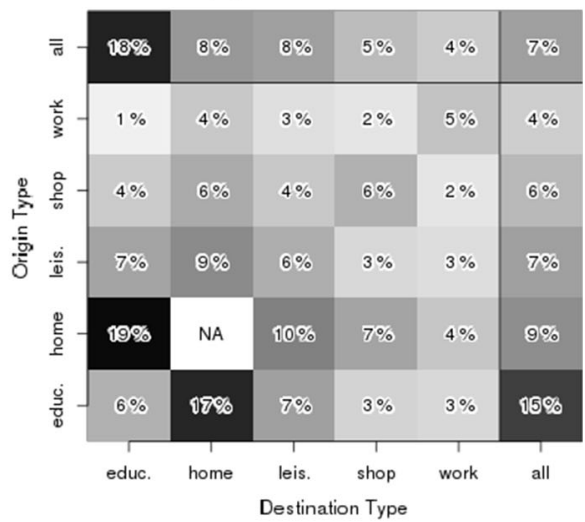

(c) National Travel Survey

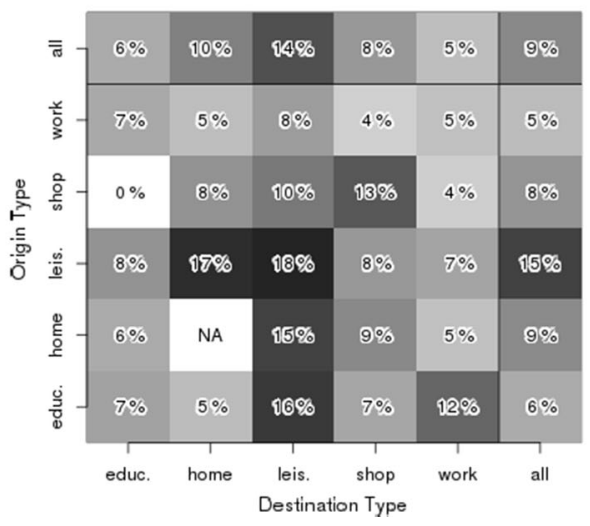

Fig. 4 Activity type to activity type "car passenger" mode shares

Overall, those results point both strengths and limitations of the current implementation. First, the framework is able to represent the emergence of joint travel, and in particular the emergence of patterns per activity type, due to the constraints specific to the household context. Though applied only to the household context here, the model is formulated in a way compatible with any social network topology, and experiments with a synthetic network of leisure contacts are in progress. However, the experiments also make clear the difficulty to apply such a model. Indeed, most differences with observed data are arguably due, at least in part, to the absence of other social contacts than household members in the scenario. Building a fully operational scenario thus requires not only to generate synthetic social networks, for instance using an approach such as the one of Arentze et al. (2012), but to calibrate behavior for those different networks: individuals are known to behave differently with different kind of social contacts (Deutsch and Goulias 2013). Finally, this approach works with exogenously given activity chains. In particular, which activity is performed or not is not part of the optimisation process. However, this kind of decision is likely to be performed jointly: only one person in the household will do grocery shopping, or the household will go as a whole to the restaurant. In the runs presented here, such processes were not included. The approach would probably benefit from co-generating the 
initial plans-which however strengthens the data requirements in the initial phase, as household-level diaries become necessary, instead of individual ones.

\section{Conclusion}

This paper presented a validation run of an approach to simulate joint decisions in the trans- portation context, using the MATSim activity-based transportation microsimulation framework. It compared the results of simulations of intra-household ride-sharing in the Zurich area, Switzerland, with travel diary data. The analysis of the results showed that the approach, in its current implementation, is able to represent the emergence of joint travel in the household context. The absence of other types of social contacts in the simulation, combined with the absence of data about which kind of social contact trips and activities were performed with in the National Travel Survey, make it difficult to assess precisely the quality of the results. To tackle this issue, experiments using a synthetic network of leisure contacts, generated using the approach of Arentze et al. (2012), are in preparation.

Considering both households and social contacts at the same time comes with new calibration challenges, as behavior is likely to vary with the kind of social contact (Deutsch and Goulias 2013).

Even with this difficulty of interpretation, the present results allow to identify directions for future work. First, in the scenario used here, the initial activity schedules of agents were sampled independently for each household member, probably leading to an underestimation of the number of possible intra-household joint leisure activities (i.e. the number of households within which several members plan a leisure activity). A way to improve the results would thus be to consider activity schedule generation at the household level. Such a model however requires even more detailed disaggregate data than the current approach for initial demand generation, whereas this kind of data is long and costly to collect-and is not readily available in Switzerland. Hence, another way to cope with this would be to represent explicitly the higher flexibility of discretionary activities in the simulation, by including or removing activities. Such a possibility was experimented with, but not validated, by Feil (2010).

Another limitation of the current implementation is that it does not include the generation of pure "serve passenger" tours, but only considers drivers picking up and dropping off passengers during their already-scheduled trips. The inclusion of such tours could be considered, depending on how the model performs when both households and social contacts are considered in the simulation.

Also, due to the lack of data, car resources in households were not considered. However, this most probably is a strong incentive for intra-household ride sharing, as pointed out by the results of Ho and Mulley (2013), and this kind of information should be included in the scenario.

Acknowledgments The work presented in this paper is part of the project "EUNOIA: Evolutive Usercentric Net- works for Intra-urban Accessibility", funded under the European Union's Seventh Framework Programme ICT Programme. 


\section{References}

Amt für Verkehr, Volkswirtschaftsdirektion Kanton Zürich.: Gesamtverkehrsmodell, webpage, March 2011, http://www.afv.zh.ch/internet/volkswirtschaftsdirektion/afv/de/home.html (2011)

Arentze, T.A., M. Kowald, Axhausen K.W.: A method to model population-wide social networks for large scale activity-travel micro-simulation. Paper presented at the 91st Annual Meeting of the Transportation Research Board, Washington, D.C., January 2012

Arentze, T.A., Timmermans, H.J.P.: A need-based model of multi-day, multi-person activity generation. Transp. Res. Part B 43(2), 251-265 (2009)

Axhausen, K.W.: Social networks and travel: Some hypotheses. In Donaghy, K. P., Pop- pelreuter, S., Rudinger G. (eds.) Social Dimensions of Sustainable Transport Transatlantic Perspectives. Ashgate, Aldershot (2005)

Axhausen, K.W.: Neue Modellansätze der Verkehrsnachfragesimulation: entwicklungslinien, Stand der Forschung, Forschungsperspektiven. Stadt Region Land 81, 149-164 (2006)

Bradley, M.A., Vovsha, P.: A model for joint choice of daily activity pattern types of household members. Transportation 32(5), 545-571 (2005)

Carrasco, J.A., Habib, K.M.N.: Understanding the social embeddedness of activity- travel participation: The case of frequency and duration of social activities. Paper presented at the 12th International Conference on Travel Behaviour Research (IATBR), Jaipur, December 2009

Carrasco, J.A., Miller, E.J., Wellman, B.: How far and with whom do people socialize? Empirical evidence about the distance between social network members. Transp. Res. Rec. 2076, 114-122 (2008)

Charypar, D., Nagel, K.: Generating complete all-day activity plans with genetic algorithms. Transportation 32(4), 369-397 (2005)

Chow, J.Y., Recker, W.W.: Inverse optimization with endogenous arrival time constraints to calibrate the household activity pattern problem. Transp. Res. Part B 46(3), 463-479 (2012)

Deutsch, K., Goulias, K.G.: Decision makers and socializers, social networks and the role of individuals as participants. Transportation 40(4), 755-771 (2013)

Dubernet, T., Axhausen, K.W.: Including joint decision mechanisms in a multiagent transport simulation. Transp. Lett. 5(4), 175-183 (2013)

Dugundji, E.R., Páez, A., Arentze, T.A.: Social networks, choices, mobility, and travel. Environ. Plan. B 35(6), 956-960 (2008)

Dugundji, E.R., Páez, A., Arentze, T.A.: Urban mobility and social-spatial contact: introduction. Environ. Plan. A 44(5), 1011-1015 (2012)

Dugundji, E.R., Páez, A., Arentze, T.A., Walker, J.L., Carrasco, J.A., Marchal, F., Nakanishi, H.: Transportation and social interactions. Transportation 45(4), 239-247 (2011)

Feil, M.: Choosing the daily schedule: expanding activity-based travel demand modelling, Ph.D. Thesis, ETH Zurich, Zurich, (2010)

Ficici, S.G.: Solution concepts in coevolutionary algorithms, Ph.D. Thesis, Brandeis University, Waltham (2004)

Ficici, S.G., Melnik, O., Pollack, J.B.: A game-theoretic and dynamical-systems analysis of selection method in coevolution. IEEE Trans. Evolut. Comput. 9(6), 580-602 (2005)

Frei, A.: Networks, geography and travel: travel between infrastructure and social structure, Ph.D. Thesis, ETH Zurich, Zurich (2012)

Gan, L.P., Recker, W.W.: A mathematical programming formulation of the household activity rescheduling problem. Transp. Res. Part B 42(6), 571-606 (2008)

Gliebe, J.P., Koppelman, F.S.: A model of joint activity participation. Transportation 1, 49-72 (2002)

Gliebe, J.P., Koppelman, F.S.: Modeling household activity-travel interactions as parallel constrained choices. Transportation 32(5), 449-471 (2005)

Golob, T.F.: A simultaneous model of household activity participation and trip chain generation. Transp. Res. Part B 34(5), 355-376 (2000)

Golob, T.F., McNally, M.G.: A model of activity participation and travel interactions between household heads. Transp. Res. Part B 31(3), 177-194 (1997)

Habib, K.M.N., Carrasco, J.A.: Investigating the role of social networks in start time and duration of activities: trivariate simultaneous econometric model. Transp. Res. Rec. 2230, 1-8 (2011)

Hackney, J.K.: Integration of social networks in a large-scale travel behavior microsimulation, Ph.D. Thesis, ETH Zurich, Zurich (2009)

Han, Q., Arentze, T.A., Timmermans, H.J.P., Janssens, D., Wets, G.: The effects of social networks on choice set dynamics: results of numerical simulations using an agent- based approach. Transp. Res. Part A 45(4), 310-322 (2011) 
Ho, C., Mulley, C.: Tour-based mode choice of joint household travel patterns on weekend and weekday. Transportation 40(4), 789-811 (2013)

Horni, A.: Destination choice modeling of discretionary activities in transport microsimulations, Ph.D. Thesis, ETH Zurich, Zurich (2013)

Kato, H., Matsumoto, M.: Intra-household interaction in a nuclear family: a utility- maximizing approach. Transp. Res. Part B 43(2), 191-203 (2009)

Liao, L., Arentze, T.A., Timmermans, H.J.P.: Multi-state supernetwork framework for the two-person joint travel problem. Transportation 40(4), 813-826 (2013)

Ma, H., Arentze, T.A., Timmermans, H.J.P.: Incorporating selfishness and altruism int dynamic joint activity-travel scheduling. Paper presented at the 13th International Conference on Travel Behaviour Research (IATBR), Toronto, July 2012

Ma, H., Ronald, N., Arentze, T.A., Timmermans, H.J.P.: New credit mechanism for semicooperative agentmediated joint activity-travel scheduling. Transp. Res. Rec. 2230, 104-110 (2011)

Meister, K.: Contribution to agent-based demand optimization in a multi-agent transport simulation, Ph.D. Thesis, ETH Zurich, Zurich (2011)

Meister, K., Balmer, M., Ciari,.F, Horni, A., Rieserm M., Waraich, R.A., Axhausen, K.W.: Large-scale agent-based travel demand optimization applied to Switzerland, including mode choice. Paper presented at the 12th World Conference on Transportation Research, Lisbon, July 2010

Meister, K., Frick, M., Axhausen, K.W.: A GA-based household scheduler. Transportation 32(5), $473-494$ (2005)

Miller, E.J., Roorda, M.J., Carrasco, J.A.: A tour-based model of travel mode choice. Transportation 32(4), 399-422 (2005)

Moore, J., Carrasco, J.A., Tudela, A.: Exploring the links between personal networks, time use, and the spatial distribution of social contacts. Transportation 40(4), 773-788 (2013)

Müller, K., Axhausen, K.W.: Using survey calibration and statistical matching to reweight and distribute activity schedules. Paper presented at the 93rd Annual Meeting of the Transportation Research Board, Washington, D.C., January 2014

Nagel, K., Flötteröd, G.: Agent-based traffic assignment: going from trips to behavioral travelers. Paper presented at the 12th International Conference on Travel Behaviour Research (IATBR), Jaipur, December 2009

Popovici, E., Wiegand, R.P., De Jong, E.D.: Coevolutionary principles. In: Rozenberg, G., Bäck, T., Kok, J.N. (eds.) Handbook of Natural Computing, pp. 987-1033. Springer, Heidelberg (2012)

Recker, W.W.: The household activity pattern problem: general formulation and solution. Transp. Res. Part B 29(1), 61-77 (1995)

Rieser, M.: Adding transit to an agent-based transportation simulation, Ph.D. Thesis, Technical University Berlin, Berlin (2010)

Ronald, N., Arentze, T.A., Timmermans, H.J.P.: Modelling social interactions between individuals for joint activity scheduling. Transp. Res. Part B 46(2), 276-290 (2012)

Schlich, R., Schönfelder, S., Hanson, S., Axhausen, K.W.: Structures of leisure travel: temporal and spatial variability. Transp. Rev. 24(2), 219-237 (2004)

Schummer, J.R., Vohra, V.: Mechanism design without money. In: Nisan, N., Roughgarden, T., Tardos, É., Vazirani, V.V. (eds.) Algorithmic Game Theory, pp. 243-266. Cambridge University Press, Cambridge (2013)

Vovsha, P., Gupta, S.: A model for work activity schedules with synchronization for multiple-worker households. Transportation 40(4), 827-845 (2013)

Vrtic, M., Fröhlich, P., Axhausen, K.W.: Schweizerische Netzmodelle für Strassen- und Schienenverkehr. In: Bieger, T., Lässer, C., Maggi, R. (eds.) Jahrbuch 2002/2003 Schweiz- erische Verkehrswirtschaft, pp. 119-140. Schweizerische Verkehrswissenschaftliche Gesellschaft (SVWG), St Gallen (2003)

Wardrop, J.G.: Some theoretical aspects of road traffic research. Proc. Inst. Civil Eng. 1(3), 325-362 (1952)

Zhang, J., Timmermans, H.J.P., Borgers, A.W.J.: A model of household task allocation and time use. Transp. Res. Part B 39(1), 81-95 (2005)

Zhang, J., Timmermans, H.J.P., Borgers, A.W.J.: Utility-maximizing model of household time use for independent, shared, and allocated activities incorporating group decision mechanisms. Transp. Res. Rec. 1807, 1-8 (2007)

Thibaut Dubernet is member of the Institute for Transport Planning and Systems (IVT) at ETH Zurich since 2011, after graduating in Urban Systems Engineering at the Technical University of Compiegne (UTC). His research focuses on the influence of inter-personal interactions on mobility behavior, with a strong focus on simulation techniques. 
Dr. K.W. Axhausen is Professor of Transport Planning at the ETH Zürich. Before he worked at the Leopold-Franzens Universität, Innsbruck, Imperial College London, the University of Oxford and the Universität Karlsruhe. He has been involved in the measurement and modelling of travel behaviour for the last 30 years contributing especially to the literature on stated preferences, micro-simulation of travel behaviour, valuation of travel time and its components, parking behaviour, activity scheduling and travel diary data collection. Current work focuses on the agent-based micro-simulation toolkit MATSim (see www.matsim.org) and on the land-use/transport interaction. 